\title{
Grand Challenges in Nanomaterial-Based Electrochemical Sensors
}

\author{
Celia Ferrag ${ }^{1}$ and Kagan Kerman ${ }^{1,2 *}$ \\ ${ }^{1}$ Department of Chemistry, University of Toronto, Toronto, ON, Canada, ${ }^{2}$ Department of Physical and Environmental \\ Sciences, University of Toronto at Scarborough, Toronto, ON, Canada
}

Keywords: electrochemical sensor, nanomaterial, real samples, stability, reproducibility, limit of detection (LOD), interference

Electrochemical sensors have an enormous potential in a wide variety of environmental, industrial, and medicinal applications. Apart from the immense success of glucose sensors, much more work is still needed in order to make electrochemical sensors have a widespread impact and application. For example, the current circumstances of the COVID-19 pandemic demonstrated the importance and urgency of having accurate and rapid diagnostic devices (Jiang et al., 2020). The advancement of sensors could truly help stop the spread of many infectious diseases (Vermisoglou et al., 2020) and detect the early onset of various illnesses such as neurodegenerative diseases (Kim et al., 2020). Compared to other diagnostic tools currently available, electrochemical sensors have many advantages such as low-cost, rapid and real-time detection with simple operation (Idili et al., 2019; Ligler and Gooding, 2019). They can also be mass-produced and miniaturized into portable devices (Li et al., 2017; Idili et al., 2019; Ligler and Gooding, 2019).

The number of research groups reporting the development of novel electrochemical sensors is growing exponentially. Most of the reported sensors have carbon- and gold-based surfaces. These surfaces are popular amongst researchers because they are stable, biocompatible, and provide good electron transfer kinetics. Unfortunately, the unmodified surfaces often lack the sensitivity and selectivity required for the electrochemical detection of trace analytes. To overcome this challenge, nanomaterials have been incorporated within the electrode surfaces (Quesada-González and Merkoçi, 2018; Muniandy et al., 2019). Nanomaterials range from 1-100 nm in size and are extremely beneficial due to the large surface-to-volume ratio and surface area (Quesada-González and Merkoçi, 2018; Muniandy et al., 2019). Modification of nanomaterials on sensor surfaces allows them to have enhanced interfacial adsorption with improved electrocatalytic activity, biocompatibility, and faster electron transfer kinetics. All these advantages give the sensor a better selectivity and sensitivity toward the detection of specific analytes as well as a superior overall performance (Quesada-González and Merkoçi, 2018; Ligler and Gooding, 2019; Muniandy et al., 2019; Jiang et al., 2020; Kim et al., 2020; Vermisoglou et al., 2020).

Recently, some of the most reported nanomaterials used in the fabrication of electrochemical sensors have been different forms of graphene, such as graphene nanoribbons (Ismail et al., 2017) and graphene flowers, which allow for good conductivity and fast electron transfer rates (Ismail et al., 2017; Quesada-González and Merkoçi, 2018; Muniandy et al., 2019; Jiang et al., 2020; Vermisoglou et al., 2020). Carbon nanotubes (CNTs) have extraordinary metallic/semiconducting properties, which significantly enhance the electrocatalytic performance of sensors (Musameh et al., 2002; Banks et al., 2006; Sljukic et al., 2006). Metallic nanoparticles and quantum dots possess remarkable conductive and optical properties that give a higher sensitivity to electrochemical sensors (Ismail et al., 2017; Ponnaiah et al., 2018; Ligler and Gooding, 2019). Although nanomaterials have been proven to be remarkably valuable in sensors, their synthesis

Front. Sens. 1:583822.

doi: 10.3389/fsens.2020.583822 as well as visual and chemical characterization often require skilled technicians to operate 
the sophisticated instrumentation. As for metallic nanoparticles and quantum dots, a common challenge is maintaining the stability of these materials, because they are prone to aggregation along with sensitivity to environmental factors such as thermal heating, UV exposure and ionic strength of aqueous media. Efforts to overcome this problem often involve the synthesis of core-shell structures and capping with stabilizing agents in order to preserve the colloid from aggregating or degrading. Similarly, researchers frequently try to control the configuration of the ligand shells to protect quantum dots from degradation (Ismail et al., 2017; Ponnaiah et al., 2018; Quesada-González and Merkoçi, 2018).

Briefly, the biggest challenges encountered in the development of electrochemical sensors can be listed as follows: (1) obtaining a low limit of detection (LOD); (2) suppressing the nonspecific adsorption of interfering species, and (3) maintaining the reproducibility and stability of the sensor in complex real matrices.

An essential property to investigate when developing an electrochemical sensor is the LOD. This value entails the lowest concentration or quantity of a specific analyte that can be reliably detected within an acceptable signal-to-noise ratio. Developing a sensor with a low LOD is crucial because often analytes exist at trace concentrations in real samples. Due to the innovation of nanomaterial-modified surfaces, LODs with values as low as picomole and femtomole levels have been achieved in the case of some ultra-sensitive sensors (Suherman et al., 2017; Li X. et al., 2018; Ponnaiah et al., 2018; Alizadeh et al., 2019; Wu et al., 2019; Gupta et al., 2020). For example, a sensor modified with open-ended CNTs was reported to have picomolar levels of sensitivity for the detection of neurotransmitters (Gupta et al., 2020). An ultrasensitive sensor for the detection of $\mathrm{Hg}^{2+}$ was developed by modification of a glassy carbon electrode with silver nanoparticles resulting in picomolar level LOD values (Suherman et al., 2017). Yet, these modified surfaces remain challenging as they are not often as reproducible as one would hope. For example, since it is difficult to control the synthesis and immobilization of nanoparticles with varying populations of size and shape, the conformation and topology of these nanomaterials might differ between each sensor (Wu et al., 2019). In addition, as mentioned previously, nanoparticles tend to alter their behavior under varying environmental conditions. The trade-off associated with increased complexity of the modified surfaces is the reproducibility issues that unfortunately arise. Sensor-to-sensor reproducibility is extremely important in the manufacturing stages since it is not feasible to test every sensor produced in mass-production facilities. Instead, once the sensors are fabricated, statistical sampling of a sub-population of the sensors is performed, and the testing and calibration results should be applicable to the entire batch.

Arguably, the most important test to confirm the validity of a sensor is the "real sample" application. If the sensor is not stable or functional in real samples then it cannot be validated as a diagnostic tool. A wide variety of real samples are often used with electrochemical sensors, the most common ones being urine, blood serum, sweat, saliva, tear, and interstitial fluid ( $\mathrm{Li}$ et al., 2017; Lipani et al., 2018; Tseng et al., 2018; de Castro et al., 2019; Idili et al., 2019; Karpova et al., 2019; Sempionatto et al., 2019; Azeredo et al., 2020; Baghelani et al., 2020). The matrix effect tends to negatively interfere with the detection of a specific analyte which lowers the recovery values and the sensitivity of the sensor. To help overcome the matrix effect, researchers frequently dilute the samples in order to shift the effect of interferences below a tolerable threshold. However, the more the sample is diluted, the further it is from reality. Ideally, sensors should be able to function effectively in pure real samples such as whole blood without any sample dilution or processing (Li et al., 2017). Saliva samples are also quite rich and complex and often need filtration or dilution to be successfully be used as a real sample (de Castro et al., 2019). For urine samples, the main challenge is the different $\mathrm{pH}$ ranges found which can impact the position of peak potential and the height of current intensity as well as cause stability issues (Azeredo et al., 2020). Tear fluid has also recently gained significant interest because it has lower complexity with easy access for non-invasive sampling techniques. Tear fluid is often used to investigate the progression of ocular diseases and diabetes (Tseng et al., 2018; Sempionatto et al., 2019). Some disadvantages with tear fluid include that the $\mathrm{pH}$ can vary, the sample volume is low and the tears from emotion and irritation may differ in composition (Tseng et al., 2018; Sempionatto et al., 2019). Real samples often contain many species that can easily adsorb onto the surface. Nonspecific adsorption has been one of the main roadblocks against utilizing electrochemical sensors in real-life applications because it tends to significantly decrease sensitivity, specificity, and reproducibility of the sensors. In the point-of-care, researchers are tackling this challenge with innovative materials and methods to improve sensor performance (Li Y. et al., 2018; Lichtenberg et al., 2019). Both passive and active methods have been used. Passive methods aim to create a hydrophilic and non-charged layer to obstruct protein adsorption on the surface by using different organic materials such as polymers. Conversely, the aim of active methods is to produce surface shear forces that are stronger than the adhesion forces of the bound non-specific biomolecules on the surface (Li Y. et al., 2018; Lichtenberg et al., 2019).

Notably, the stability of sensors has also been a challenge limiting their applications in remote locations under varying temperature conditions. Sensors are often characterized by their shelf-life; hence, it is important to develop sensors that can work for a relatively long period of time. When using nanomaterials, long-term stability might become a major concern due to issues related to aggregation and flaking of nanomaterialmodified layers. Recently, incorporation of sol-gel materials and ceramics along with nanomaterials has been proven to increase the stability of sensors (Kim et al., 2018; Li et al., 2019; Meng et al., 2020). Moreover, designing sensors that are stretchable, self-healing, water-processable and wearable has recently been a major advancement in the sensing field (Kim et al., 2018; Li et al., 2019; Meng et al., 2020). These types of miniaturized sensors, which have great stability, are often made with rubber-like composites, hydrogels, organogels and novel polymers. Combining these highly stretchable materials with the excellent electrical conductivity of nanomaterials makes 
for remarkable sensors with superior analytical performance characteristics (Kim et al., 2018; Li et al., 2019; Meng et al., 2020).

Electrochemical sensors have demonstrated a great potential for the future of diagnostic tools. However, the challenges outlined above, not only apply for clinical applications, but also across other fields such as environmental monitoring, food safety, forensic analysis, agriculture, defense, and military applications as well as electronics industry. Fortunately, the breakthrough created through the applications of nanomaterials represents a significant advancement that impacts across all the fields mentioned above. To continue advancing in the right direction, it is important for researchers to keep on developing innovative solutions in order to introduce more smart sensors in our everyday life.

\section{REFERENCES}

Alizadeh, T., Atashi, F., and Ganjali, M. R. (2019). Molecularly imprinted polymer nano-sphere/multi-walled carbon nanotube coated glassy carbon electrode as an ultra-sensitive voltammetric sensor for picomolar level determination of RDX. Talanta 194, 415-421. doi: 10.1016/j.talanta.2018.10.040

Azeredo, N. F. B., Gonçalves, J. M., Rossini, P. O., Araki, K., Wang, J., and Angnes, L. (2020). Uric acid electrochemical sensing in biofluids based on $\mathrm{Ni} / \mathrm{Zn}$ hydroxide nanocatalyst. Microchim. Acta 187, 1-11. doi: 10.1007/s00604-020-04351-2

Baghelani, M., Abbasi, Z., Daneshmand, M., and Light, P. E. (2020). Noninvasive continous glucose monitoring system using a chipless printable sensor based on split ring microwave resonators. Sci. Rep. 10:12980. doi: 10.1038/s41598-020-69547-1

Banks, C., E., Crossley, A., Salter, C., and Wilkins, S. J., Compton, R. G. (2006). Carbon nanotubes contain metal impurities which are responsible for the "electrocatalysis" seen at some nanotube-modified electrodes. Angew. Chem. Int. Ed. 45, 2533-2537. doi: 10.1002/anie.200600033

de Castro, L. F., de Freitas, S. V., Duarte, L. C., de Souza, A. C. J., Paixão, T. R., and Tomazelli Coltro, W. K. (2019). Salivary diagnostics on paper microfluidic devices and their use as wearable sensors for glucose monitoring. Anal. Bioanal. Chem. 411, 4919-4928. doi: 10.1007/s00216-019-01788-0

Gupta, P., Tsai, K., Ruhunage, C. K., Gupta, V. K., Rahm, C. E., Jiang, D., et al. (2020). True picomolar neurotransmitter sensor based on open-ended carbon nanotubes. Anal. Chem. 92, 8536-8545. doi: 10.1021/acs.analchem.0c01363

Idili, A., Arroyo-Curras, N., Ploense, K. L., Csordas, A. T., Kuwahara, M., Kippin, T. E., et al. (2019). Seconds-resolved pharmacokinetic measurements of the chemotherapeutic irinotecan in situ in the living body. Chem. Sci. 10, 8164-8170. doi: 10.1039/C9SC01495K

Ismail, N. S., Hoa, L. Q., Huong, V. T., Inoue, Y., Yoshikawa, H., Saito, M., et al. (2017). Electrochemiluminescence based enzymatic urea sensor using nanohybrid of isoluminol-gold nanoparticle-graphene oxide nanoribbons. Electroanalysis 29, 938-943. doi: 10.1002/elan.201600477

Jiang, Z., Feng, B., Xu, J., Qing, T., Zhang, P., and Qing, Z. (2020). Graphene biosensors for bacterial and viral pathogens. Biosens. Bioelectron. 166:112471. doi: 10.1016/j.bios.2020.112471

Karpova, E. V., Shcherbacheva, E. V., Galushin, A. A., Vokhmyanina, D. V., Karyakina, E. E., and Kayrakin, A. A. (2019). Noninvasive diabetes monitoring through continuous analysis of sweat using flow-through glucose biosensor. Anal. Chem. 91, 3778-3783. doi: 10.1021/acs.analchem.8b0 5928

Kim, J., Jeerapan, I., Sempionatto, J. R., Barfidokht, A., Mishra, R. K., Campbell, A. S., et al. (2018). Wearable bioelectronics: enzyme-based body-worn electronic devices. Acc. Chem. Res. 51, 2820-2828. doi: 10.1021/acs.accounts.8b00451

Kim, K., Lee, C. H., and Park, C. B. (2020). Chemical sensing platforms for detecting trace-level Alzheimer's core biomarkers. Chem. Soc. Rev. 49, 5446-5472. doi: 10.1039/D0CS00107D

\section{AUTHOR CONTRIBUTIONS}

CF wrote the original draft of the manuscript. KK revised and edited the manuscript. All authors contributed to the article and approved the submitted version.

\section{ACKNOWLEDGMENTS}

KK acknowledges financial support from the Canada Research Chair Tier-2 award for Bioelectrochemistry of Proteins (Project no. 950-231116), Ontario Ministry of Research and Innovation (Project no. 35272), Discovery Grant (Project no. 3655) from Natural Sciences and Engineering Research Council of Canada (NSERC), and Canada Foundation for Innovation (Project no. 35272).

Li, H., Dauphin-Ducharme, P., Ortega, G., and Plaxco, K. W. (2017). Calibrationfree electrochemical biosensors supporting accurate molecular measurements directly in undiluted whole blood. J. Am. Chem. Soc. 139, 11207-11213. doi: 10.1021/jacs.7b05412

Li, T., Li, Y., and Zhang, T. (2019). Materials, structures, and functions for flexible and stretchable biomimetic sensors. Acc. Chem. Res. 52, 288-296. doi: 10.1021/acs.accounts.8b00497

Li, X., Peng, G., Cui, F., Qiu, Q., Chen, X., and Huang, H. (2018). Double determination of long noncoding RNAs from lung cancer via multi-amplified electrochemical genosensor at sub-femtomole level. Biosens. Bioelectron. 113, 116-123. doi: 10.1016/j.bios.2018.0 4.062

Li, Y., Xu, Y., Fleischer, C. C., Huang, J., Lin, R., Yang, L., et al. (2018). Impact of anti-biofouling surface coatings on the properties of nanomaterials and their biomedical applications. J. Mater. Chem. B. 6, 9-24. doi: 10.1039/C7TB01695F

Lichtenberg, J. Y., Ling, Y., and Kim, S. (2019). Non-specific adsorption reduction methods in biosensing. Sensors 19:2488. doi: 10.3390/s19112488

Ligler, F. S., and Gooding, J. J. (2019). Lighting up biosensors: now and the decade to come. Anal. Chem. 91, 8732-8738. doi: 10.1021/acs.analchem.9b00793

Lipani, L. B., Dupont, G. R., Doungmene, F., Marken, F., Tyrell, R. M., Guy, R. H., et al. (2018). Non-invasive, transdermal, path-selective and specific glucose monitoring via a graphene-based platform. Nat. Nanotechnol. 13, 504-511. doi: 10.1038/s41565-018-0112-4

Meng, L., A., and Turner, P. F., Mak, W. C. (2020). Soft and flexible material-based affinity sensors. Biotechnol. Adv. 39:107398. doi: 10.1016/j.biotechadv.2019.05.004

Muniandy, S., The, S. J., Thong, K. L., Thiha, A., Dinshaw, I. J., Lai, C. W., et al. (2019). Carbon nanomaterial-based electrochemical biosensors for foodborne bacterial detection. Crit. Rev. Anal. Chem. 49, 510-533. doi: 10.1080/10408347.2018.156 1243

Musameh, M., Wang, J., Merkoci, A., and Lin, Y. (2002). Low-potential stable NADH detection at carbon-nanotube-modified glassy carbon electrodes. Electrochem. Commun. 4, 743-746. doi: 10.1016/S1388-2481(02)00451-4

Ponnaiah, S. K., Periakaruppan, P., and Vellaichamy, B. (2018). New electrochemical sensor based on a silver-doped iron oxide nanocomposite coupled with polyaniline and its sensing application for picomolar-level detection of uric acid in human blood and urine samples. J. Phys. Chem. B. 122, 3037-3046. doi: 10.1021/acs.jpcb.7b1 1504

Quesada-González, D., and Merkoçi, A. (2018). Nanomaterial-based devices for point-of-care diagnostic applications. Chem. Soc. Rev. 47, 4697-4709. doi: 10.1039/C7CS00837F

Sempionatto, J. R., Brazaca, L. C., Garcia-Carmona, L., Bolat, G., Campbell, A. S., Martin, A., et al. (2019). Eyeglasses-based tear biosensing system: non-invasive detection of alcohol, vitamins and glucose. Biosens. Bioelectron. 137, 161-170. doi: 10.1016/j.bios.2019.04.058 
Sljukic, B., Banks, C. E., and Compton, R. G. (2006). Iron oxide particles are the active sites for hydrogen peroxide sensing at multiwalled carbon nanotube modified electrodes. Nano Lett. 6, 1556-1558. doi: 10.1021/nl060366v

Suherman, A. L., Ngamchuea, K., Tanner, E. E. L., Sokolov, S. V., Holter, J., Young, Y. P., et al. (2017). Electrochemical detection of ultratrace (picomolar) levels of $\mathrm{Hg}^{2+}$ using a silver nanoparticle-modified glassy carbon electrode. Anal. Chem. 89, 7166-7173. doi: 10.1021/acs.analchem.7b01304

Tseng, R. C., Chen, C. C., Hsu, S. M., and Chuang, H. S. (2018). Contact-lens biosensors. Sensors 18:2651. doi: 10.3390/s1808 2651

Vermisoglou, E., Panacek, D., Jayaramulu, K., Pykal, M., Frebort, I., Kolar, M., et al. (2020). Human virus detection with graphene-based materials. Biosens. Bioelectron. 166:112436. doi: 10.1016/j.bios.2020.11 2436
Wu, Y., Tilley, R. D., and Gooding, J. J. (2019). Challenges and solutions in developing ultrasensitive biosensors. J. Am. Chem. Soc. 141, 1162-1170. doi: 10.1021/jacs.8b09397

Conflict of Interest: The authors declare that the research was conducted in the absence of any commercial or financial relationships that could be construed as a potential conflict of interest.

Copyright (๑) 2020 Ferrag and Kerman. This is an open-access article distributed under the terms of the Creative Commons Attribution License (CC BY). The use, distribution or reproduction in other forums is permitted, provided the original author(s) and the copyright owner(s) are credited and that the original publication in this journal is cited, in accordance with accepted academic practice. No use, distribution or reproduction is permitted which does not comply with these terms. 